\title{
Der Begriff der Notwendigkeit in der Antike und in der Gegenwart
}

\section{Einige Befunde}

(1.) Heraklit, DK A 8: Für Heraklit fallen zusammen: ananke, heimarmene, logos. Dasselbe gilt von den Stoikern. ${ }^{1}$

(2.) Platon, Timaios, 48 a (p. $82 \mathrm{f}$ der angegebenen Ausgabe):

"Denn das Werden dieser Weltordnung entstand als aus einer Vereinigung von Notwendigkeit und Vernunft gemischt. Indem aber die Vernunft über die Notwendigkeit dadurch herrschte, daß sie sie überredete, das meiste des im Entstehen Begriffenen dem Besten entgegenzuführen, so bildete sich auf diese Weise und indem die Notwendigkeit durch besonnene Überredung besiegt wurde, am Anfang dieses All."

Parallel dazu: Platon, Philebos, 28d (p. 310 der angg. Ausgabe): [Sokrates:] "Ob wir wohl, o Protarchos, sagen wollen, über alles insgesamt und über dies sogenannte Ganze walte die Gewalt des Vernunftlosen und des Zufälligen und das Ohngefähr, oder im Gegenteil, wie auch unsere Vorfahren gesagt haben, Vernunft und eine wundervolle anordnende Einsicht beherrsche alles?" (Übersetzung modifiziert.)

(3.) Ein Echo der Timaios-Stelle findet sich bei Plotin, Enn. III, 2, 2, 31-36 (p. 47 der angg. Ausgabe):

"Dies unser All ist ja nicht wie das dort droben Geist und rationale Form, sondern es hat nur Anteil an Geist und rationaler Form.

\footnotetext{
${ }^{1}$ Vgl. hierzu: Historisches Wörterbuch der Philosophie, Bd. 6, "Notwendigkeit", p. 946, p. 953.
}

Possibility and Reality - Metaphysics and Logic, eds. H. Rott and V. Horák, Ontos Verlag, Frankfurt a.M., London 2003, pp. 13-50. 
Deswegen bedurfte es auch einer harmonischen Fügung, in der sich zusammenschlossen 'Geist und Notwendigkeit', wobei die Notwendigkeit, als vernunftlose, zum Niederen hinzieht und zum Vernunftwidrigen treibt, dennoch aber 'der Geist über die Notwendigkeit gebietet'."

(4.) Aristoteles, Met.,V, 5 (p. $190 \mathrm{f}$ der angg. Ausgabe):

Aristoteles unterscheidet (1) das Notwendige, das lebensnotwendig ist (wie Atmen und Nahrungsaufnahme), (2) das Notwendige, das notwendig ist, um ein gewisses Ziel zu erreichen (wie Arznei zu trinken, oder nach Aigina zu segeln), (3) das Notwendige, das "das Erzwungene und der Zwang [ist], welches uns gegen unsre eigene Neigung und unseren Entschluß bindet und hemmt. Denn man nennt das Erzwungene notwendig, weshalb es auch schmerzlich ist, wie Euenos sagt: 'Denn alles Notwendige ist ein betrübendes Ding.' Und der Zwang ist eine Art Notwendigkeit, wie ja Sophokles sagt: '... Es nötigt mich der Zwang zu solcher Tat.' Auch gilt die Notwendigkeit für etwas Unerbittliches, und mit Recht; denn sie steht der Bewegung nach Vorsatz und Überlegung entgegen." (1015 a 26 - 33) Schließlich ist bei Aristoteles (4) das Notwendige das, was sich nicht anders verhalten kann: "Und auf diese Bedeutung von Notwendigkeit kommen gewissermaßen alle anderen Bedeutungen desselben zurück; denn einmal von dem Erzwungenen sagt man, daß es notwendig dies tun oder leiden müsse, in dem Falle, wenn es wegen des Zwingenden nicht seiner eigenen Neigung folgen kann, indem man als Notwendigkeit das ansieht, wodurch ihm unmöglich ist, sich anders zu verhalten. Und bei den Mitursachen des Lebens und des Guten verhält es sich ebenso; denn wenn hier das Leben, dort das Gute ohne gewisse Dinge nicht möglich ist, so sind diese notwendig und diese Ursachen eine Art von Notwendigkeit." (1015 a $35-1015$ b 6) 


\section{Ein begriffliches Schema und der archaische Begriff der Notwendigkeit}

Die wohlbekannte aristotelische Unterscheidung der Vier Ursachen in Materialursache, Formursache, effiziente Ursache und Zweckursache (vgl. Met. V, 2, 1013 a 24 - 34) läßt sich zur Erhellung der im vorausgehenden Abschnitt angeführten Befunde mit Gewinn transformieren in eine Unterscheidung der Vier Notwendigkeiten: Materie-Notwendigkeit als durch die Materie gegebene Notwendigkeit, Form-Notwendigkeit als durch die Form gegebene Notwendigkeit (Notwendigkeit aufgrund eines Soseins), Effizienz-Notwendigkeit als durch das Agens gegebene Notwendigkeit (Notwendigkeit aufgrund einer ausgeübten Macht), Zweck-Notwendigkeit als durch den Zweck gegebene Notwendigkeit (Notwendigkeit für einen Zweck). Von diesen vier Notwendigkeiten spricht Aristoteles in der oben zitierten Passage drei mehr oder weniger explizit an: (1) die Form-Notwendigkeit (in der besonderen Gestalt des Lebensnotwendigen), (2) die Zweck-Notwendigkeit (in der besonderen Gestalt des für Zwecke des Alltags Notwendigen) und (3) die Effizienz-Notwendigkeit (in der besonderen Gestalt des sich gegen die eigene Neigung Durchsetzenden, des Zwangs). Aristoteles' oben angeführte vierte Notwendigkeit ist dagegen weniger eine Notwendigkeit neben den anderen von ihm genannten als eine Gemeinsamkeit aller von ihm angeführten Notwendigkeiten, etwas, das zu deren Logik gehört, nämlich ihre Verbindung zur Möglichkeit, die darin besteht, daß das in der oder der Weise Notwendige eben das ist, das nicht nicht-sein kann, sei es auch das, was unter einer gewissen Bedingung nicht nicht-sein kann: wenn eine Zuständlichkeit (Sosein) aufrechterhalten wird, oder wenn ein Zweck erfüllt wird, wenn eine Machtausübung vorliegt, oder schließlich nun auch, wenn aus Voraussetzungen logisch gefolgert wird, die erkanntermaßen nicht nichtsein können, denn Aristoteles nennt als weitere Notwendigkeit die (schlechthinnige) Beweisnotwendigkeit (Met. 1015 b 6 -9).

Die Beweisnotwendigkeit ist als maximal rationale Notwendigkeit anzusehen. Sie ist aber eine der Notwendigkeiten, die sich 
in das eben angegebene Vier-Notwendigkeiten-Schema nicht oder nicht ohne weiteres einpassen lassen $;^{2}$ eventuell ließe sie sich - so, wie Aristoteles sie meinte - als Abkömmling der Form-Notwendigkeit betrachten. ${ }^{3}$ In den beigebrachten Zitaten von Platon und Plotin ist demgegenüber, gewissermaßen am anderen Ende des Spektrums der Rationalität, unter dem Titel "Notwendigkeit" offenbar von der Materie-Notwendigkeit die Rede; diese Notwendigkeitsform wird von ihnen als eine rationalitätsabgewandte, vernunftlose, anarchische betrachtet. Als solche steht sie ganz in der Nähe des (ontologischen) Zufalls (der tyche), und insofern könnte man sagen, daß die Materie-Notwendigkeit keine eigentliche Notwendigkeit ist, denn Notwendigkeit und Zufall sind, eigentlich (für unser Begriffsempfinden), entgegengesetzt. Doch hat eben die Materie-Notwendigkeit, wie alle Notwendigkeitsbegriffe, auch ein für Notwendigkeitsbegriffe adäquates Zentralmerkmal: Alles, was materie-notwendig ist, ist unbeherrschbar, sei es epistemisch, oder im Handeln.

Die mit der Materie-Notwendigkeit festverknüpften Vorstellungen des Unbeherrschbaren, Arationalen, an sich (nach menschlichen Maßen) Ordnungslosen und auch Undurchschaubaren werden nun aber auch - wie schon immer, so auch insbesondere in der Antike - mit der Notwendigkeit des Schicksals, der heimarmene, des fatum verbunden. Gleichbedeutend stehen daneben Konnotationen der Schicksalsnotwendigkeit, die von der täterrelationalen Effizienz-Notwendigkeit herrühren, nämlich die Konnotationen des Zwingens und Beherrschens, der unwiderstehlichen Kraft; nicht zufälligerweise, demnach, wird das Schicksal oftmals personifiziert. Das notwendigkeitsadäquate Zentralmerkmal jedoch der Effizienz-Notwendigkeit selbst, nicht bloß eine mit ihr verbundene Konnotation wie bei der Schicksalsnotwendigkeit, ist eben die Unwiderstehbarkeit: Alles, was effizienz-notwendig ist, ist unwiderstehbar; die

\footnotetext{
${ }^{2}$ Eine andere derartige Notwendigkeit wird mit dem Beispiel (2) in Abschnitt 6 angesprochen.

${ }^{3}$ Dies wird in den Abschnitten 6 und 7 deutlicher werden. Axiome nach Aristoteles könnten als Ausdruck von gewissen Formen, nämlich grundgelegten intrinsischen Gesetzen gelten, und die Theoreme als Ausdruck von Sachverhalten, die durch die erstgenannten Formen (via logische Ableitung) notwendig sind.
} 
Effizienz-Notwendigkeit zwingt, sofern man sich ihr entgegenstellt.

Unbeherrschbarkeit und Unwiderstehbarkeit sind schließlich zusammen die zentralen Merkmale des archaischen Notwendigkeitsbegriffs (soweit er für uns rekonstruierbar ist), der mit dem Schicksalsbegriff verbunden und mit der archaischen Schicksalsnotwendigkeit zu identifizieren ist. Hinzukommt, kaum weniger zentral, das epistemologische und rationalitätstheoretische Merkmal der Unverstehbarkeit und Unerklärbarkeit.

\section{Rationale Notwendigkeit}

Bei Heraklit und dann vor allem bei den Stoikern und Epikureern nimmt aber die Schicksalsnotwendigkeit ein Charakteristikum an, das zu diesem letztgenannten Merkmal, der Unverstehbarkeit und Unerklärbarkeit, nicht paßt, nämlich das der rationalen, also erkennbaren und ordnungsstiftenden Gesetzlichkeit. Dieses letztere Charakteristikum ist nun an zentraler Stelle eigentümlich für die Form-Notwendigkeit: Alles, was form-notwendig ist, ist gesetzlich; d. h. was form-notwendig ist, unterliegt einem Gesetz, also nichts anderem als einer gewissen Bedingungen genügenden (weit aufgefaßten) Form oder Gestalt (eben der Form, durch die es, das Form-Notwendige, gefordert wird). Für das Bewußtsein jedenfalls der Philosophen transformiert die erkennbare Gesetzlichkeit das unverstehbare und unerklärliche Schicksal in eine verstehbare und erklärbare, unverbrüchliche Weltordnung, eben einen logos. So spricht Marc Aurel, Echo der Stoiker, vom

"logos, der das ganze Sein durchzieht und durch alle Ewigkeit hindurch in Zeitabschnitten das All verwaltet." (Pros seauton, V, 32; p. 116 der angg. Ausgabe; eigene Übersetzung.)

Und Lukrez, Echo der Epikureer, spricht zwar nicht vom logos, wohl aber vom "Sieger" (Epikur), der uns von seinem Erkenntniszug durchs kühn für erkennbar gehaltene All das Wissen davon zurückbringt,

"was entstehen kann, und was nicht, außerdem auf welche Weise denn jedem die Macht begrenzt sei und der Grenzstein tief haf- 
tend." (De rerum natura, I, 75-77; p. 12 der angg. Ausgabe; eigene Übersetzung.)

Wobei der Gedanke einer rationalen, nämlichen quasi-rechtlichen, Weltordnung - um auf das andere Ende der antiken Geistesgeschichte zu blicken - schon im berühmten Fragment des Anaximander, DK B 1, vorgebildet ist:

"Woraus aber das Werden ist den Seienden, dorthin ist auch das Vergehen, nach der Notwendigkeit. Denn sie geben einander Sühne wegen ihrer Ungerechtigkeit nach der Ordnung der Zeit." (Eigene Übersetzung.)

Zudem: Die Universalität (der logos durchzieht das ganze Sein [Marc Aurel], jedem ist die Macht begrenzt [Lukrez], woraus das Werden ist den Seienden, also allen, dorthin ist auch ihr Vergehen [Anaximander]), die mit einer solchen in Gesetzen bestehenden Weltordnung per se verbunden ist, scheint bemerkenswerterweise von Anfang an zu den Konnotationen gehört zu haben, die mit Notwendigkeit und Schicksal verknüpft wurden: Schon den Ältesten schien alles von diesen beherrscht. ${ }^{4}$

Da die Rationalisierung der Schicksalsnotwendigkeit in der Aufnahme von Konnotationen besteht, die von der wesenhaft apersonalen Form-Notwendigkeit ausgehen, ist es natürlich, daß mit jener Rationalisierung eine Entpersonalisierung verbunden ist. Das bedeutet, daß die von der Effizienz-Notwendigkeit herrührenden Konnotationen, die für die Personifizierung des Schicksals sorgten, ganz zurücktreten. Verschwunden sind natürlich die arationalen Konnotationen - bis auf gewisse Spuren, die etwa ersichtlich sind in den Worten des Heraklit, daß die Natur sich zu verbergen liebe und ein Haufen aufs Geratewohl hingeschütteter Dinge die schönste Weltordnung sei (DK B 123, B 124). Bei aller Wandlung ist aber der logos, die rationalisierte heimarmene, nach wie vor, nur etwas "lei-

\footnotetext{
${ }^{4}$ Das muß noch nicht heißen, daß sie Deterministen waren: Daraus, daß alles der Notwendigkeit unterliegt, folgt noch nicht ohne weiteres, daß alles in jeder Hinsicht der Notwendigkeit unterliegt. Und doch lag die Verknüpfung der Notwendigkeit mit dem Determinismus, mit ihrer superlativen Universalität, von Anfang an nahe. Entsprechende Überlegungen gelten für Schicksal und Fatalismus.
} 
ser" sozusagen, charakterisiert durch Unbeherrschbarkeit und Unwiderstehbarkeit. Es träfe das "Leisersein" dieser Charakteristika, wenn man statt von Unbeherrschbarkeit und Unwiderstehbarkeit nun spräche von Unverfügbarkeit und Unverbrüchlichkeit.

In dem in Abschnitt 1 angeführten Zitat aus Platons Philebos ist von der Vernunft (nous) und anordnenden Einsicht (phronesis syntattousa) die Rede, die alles beherrschen (das griechische Verb ist diakybernan). Platon hat offenbar an dieser Stelle, freilich ohne das Wort zu verwenden, eine ganz andere Notwendigkeit im Sinn als diejenige ist, die er in der schon besprochenen Timaios-Stelle meinte, wo allerdings auch schon, freilich in etwas anderer Weise, von der herrschenden Vernunft die Rede ist, nämlich von der Vernunft, die die Notwendigkeit beherrscht (das griechische Verb ist archein), merkwürdigerweise durch gutes Zureden. Stärker als "die Notwendigkeit" kann doch nur eine andere Notwendigkeit sein, nun eine Notwendigkeit, die alles zum Besten ausrichtet. Im Gleichklang mit dieser zweiten, ganz und gar rationalen platonischen Notwendigkeit führte man auch in der nichtchristlichen Antike - und vielleicht schon in den ältesten Zeiten, denn Platons Sokrates spricht von "unseren Vorfahren" - die Rationalisierung der Schicksalsnotwendigkeit auch so weit, daß mit ihr Konnotationen verbunden wurden, die von der Zweck-Notwendigkeit ausgehen: Was das Schicksal verordnete, war als solches notwendig für einen gewissen Zweck, zwar nicht für den Endzweck der Welt (der lag bei einer zyklischen Geschichtsauffassung fern), ${ }^{5}$ sondern vielmehr für den Nutzen des Ganzen. So lesen wir wiederum bei Marc Aurel:

"Es kommt aber herbei das Notwendige und dem ganzen Kosmos Zuträgliche, von dem du ein Teil bist." (Pros seauton, II, 3; p. 28 der angg. Ausgabe; eigene Übersetzung.)

In dieser weitergehenden Rationalisierung erfährt die heimarmene noch einmal eine Transformation, nämlich die Transforma-

\footnotetext{
${ }^{5}$ Sucht man nach einer Rationalisierung der Schicksalsnotwendigkeit, die dabei doch noch Schicksalsnotwendigkeit bleibt und nicht zur Schickung Gottes mutiert, durch einen als erkennbar angenommenen Endzweck der Welt (oder der Weltgeschichte), dem sie dient, so findet man derartiges erst sehr viel später, nämlich, nach dem Durchgang durch das Christentum, im Effekt bei Hegel.
} 
tion zur pronoia, wodurch ein Effekt wieder teilweise rückgängig gemacht wird, der mit ihrer ersten Transformation, der Transformation in den logos, verbunden war: die Entpersonalisierung. Die pronoia ebenso wie die archaische heimarmene - also dasjenige Schicksalskonzept, das vor dem Einsetzen des philosophischen Geistes allein gegegeben war - ist für eine Personifizierung deshalb geeignet, weil sowohl Zweckgerichtetheit, die die bei der pronoia hervorstechende Konnotation ist, als auch Effizienz, die eine der zentralen Konnotationen der archaischen heimarmene ist, aber bei der pronoia keinesfalls fehlt, beide - zumindest prima facie - auf einen personalen Träger verweisen. Marc Aurel schreibt:

"Alles, was geschieht, geschieht zu Recht. ...Ich sage nicht nur, daß es folgerichtig [ $\rightarrow$ form-notwendig], sondern auch daß es rechtmäßig [ $\rightarrow$ zweck-notwendig] ist, und so geschieht, als ob es auf Veranlassung eines höheren Wesens geschähe $[\rightarrow$ quasi effizienz-notwendig], das alles zuteilt, wie es sich nach dem Wert gehört." (Pros seauton, IV, 10; p. 68 der angg. Ausgabe; eigene Übersetzung.)

Noch deutlicher ist der personale Bezug der pronoia in der folgenden Passage, die aus demselben Textstück stammt wie das vorletzte Marc-Aurel-Zitat (diesem unmittelbar vorausgeht):

"Was von den Göttern ist, ist von Vorsehung gesättigt. Was vom Zufall ist, ist nicht ohne Natur oder Verkettung und Verknüpfung mit dem durch Vorsehung Verwalteten. Alles fließt von dort." (Pros seauton, II, 3; p. 28 der angg. Ausgabe; eigene Übersetzung.)

Nicht zufällig wird "die Vorsehung", pronoia, lateinisch providentia, zu einem der christlichen Gottesnamen. Für Boethius, am Ende der beschriebenen Rationalisierungsentwicklung, stellen providentia (nämlich die providentia des persönlichen Gottes) und fatum nur zwei Gegebenheitsweisen desselben dar: Die universale vernünftige Ordnung der Welt überzeitlich festgelegt ist (göttliche) providentia, diese Ordnung sich innerzeitlich realisierend ist fatum:

"[D]ie Vorsehung ist jene im höchsten Herrscher aller Dinge selber begründete göttliche Vernunft, die alles ordnet; das Schicksal aber die den beweglichen Dingen anhaftende planmäßige Anlage, 
durch welche die Vorsehung mit ihren Ordnungen alles verknüpft. Die Vorsehung umfaßt nämlich alles gleichmäßig, wie verschieden, wie unbegrenzt es sei, das Schicksal aber treibt das Einzelne zur Bewegung, das nach Ort, Form, Zeit verteilt ist, so daß diese Entwicklung der zeitlichen Ordnung, im Überblick des göttlichen Geistes vereinigt, Vorsehung ist, eben diese Vereinigung aber in der Zeit verteilt und entwickelt, Schicksal genannt wird." (Consolatio philosophiae, IV, p. 242 der angg. Ausgabe.)

\section{Der Reichtum der antiken Notwendigkeitskonzep- tionen}

Die vorgestellten vier Quellpunkte antiker Notwendigkeitskonzeptionen lassen sich wie folgt übersichtlich zusammenstellen:

Materie-Notwendigkeit, nicht persona-konnotativ und nicht ratiokonnotativ.

Zentralmerkmal: Unbeherrschbarkeit.

Form-Notwendigkeit, nicht persona-konnotativ, aber ratio-konnotativ.

Zentralmerkmal: Gesetzlichkeit (Form-Erforderlichkeit). Effizienz-Notwendigkeit, persona-konnotativ, aber nicht ratio-konnotativ.

Zentralmerkmal: Unwiderstehbarkeit. Zweck-Notwendigkeit, persona-konnotativ und ratio-konnotativ.

Zentralmerkmal: Zweckhaftigkeit (Zweck-Erforderlichkeit). Die Schicksalsnotwendigkeit in der Geistesgeschichte der Antike ist, wie oben deutlich geworden ist, ein konnotativ mehrdimensionaler und sich wandelnder Begriff. Das hierzu oben Gesagte läßt sich wie folgt veranschaulichend zusammenfassen:

1. ARCHAISCHE SCHICKSALSNOTWENDIGKEIT: UNBEHERRSCHBARKEIT, UnWIDERSTEHBARKEIT, Personalität, Arationalität. 
2. RATIONALISIERTE SCHICKSALSNOTWENDIGKEIT: Unbeherrschbarkeit (Unverfügbarkeit), Unwiderstehbarkeit (Unverbrüchlichkeit), GesetzlichKeIt, Apersonalität, RATIONALITÄT.

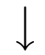

3. WEITER RATIONALISIERTE SCHICKSALSNOTWENDIGKEIT: Unbeherrschbarkeit (Unverfügbarkeit), Unwiderstehbarkeit (Unverbrüchlichkeit), Gesetzlichkeit, ZwECKHAFTigkeit, Personalität, Rationalität.

Die Reihenfolge 1. - 3. stellt hier die grob feststellbare zeitliche Reihenfolge der mit der Schicksalsnotwendigkeit verbundenen konnotativen Komplexe dar (die Pfeile sollen die zeitliche Folge veranschaulichen). Diese Reihenfolge ist nur grob feststellbar und nur im großen und ganzen richtig; denn der konnotative Komplex 3 ist für Platon nicht weniger einschlägig als für Marc Aurel, und der konnotative Komplex 1 hörte in der Antike - selbst nach dem Sieg des Christentums - nie ganz auf, in der Geisteswelt der Menschen weiterzubestehen, ja setzte sich, modifiziert, in der Gestalt des Fortuna-Konzepts selbst im Mittelalter noch fort. Die Verwendung von Großbuchstaben oder aber, im Gegenteil, von kleinen Buchstaben gibt hier die relative Prominenz der mit der Schicksalsnotwendigkeit suksessive verbundenen Konnotationen in ihren jeweiligen Komplexen wieder. Wie unschwer erkennbar ist, speisen sich die Konnotationskomplexe aus den vier bezeichneten Quellpunkten der antiken Notwendigkeitskonzeptionen.

Neben jenen vier reinen Notwendigkeiten und der zunehmend "synkretistischen" Schicksalsnotwendigkeit kannte die Antike, spätestens in Anschluß an Aristoteles, auch die Beweisnotwendigkeit, die wir schon angesprochen haben, und schließlich eine Notwendigkeit, die Aristoteles in Met. V, 5 wie folgt einführt:

"Das Notwendige nun hat entweder die Ursache der Notwendigkeit in einem anderen oder nicht in einem anderen, sondern es 
kommt vielmehr um seinetwillen anderem Notwendigkeit zu. Im ersten und strengsten Sinne notwendig ist also das Einfache; denn dies kann sich nicht auf mehrfache Weise verhalten, also auch nicht so und anders, denn dann verhielte es sich schon auf mehrfache Weise. Gibt es also gewisse ewige und unveränderlich Dinge, so findet in ihrer Notwendigkeit kein Zwang und kein Gegensatz gegen die Natur statt." (1015 b 9-15)

Aristoteles unterscheidet hier offenbar die extrinsische oder relationale Notwendigkeit, wo, in einem gegebenen Fall, die einschlägige Ursache, oder besser: der einschlägige Grund des Notwendigseins des vorliegenden Notwendigen in einem anderen als es selbst ist, von der intrinsischen Notwendigkeit, bei der der einschlägige Grund des Notwendigseins des vorliegenden Notwendigen nicht in einem anderen, sondern in ihm selbst (in dem einschlägigen Aspekt von ihm) ist, wobei Aristoteles die intrinsische Notwendigkeit, Notwendigkeit "im ersten und strengsten Sinne", den einfachen Dingen, d. h. den ewigen und unveränderlichen (bzw. deren Verhalten) zuschreibt. Obwohl letzteres - die augenscheinliche Fixierung der intrinsischen Notwendigkeit auf einen bestimmten Gegenstandsbereich, ihre augenscheinliche Identifizierung mit der Notwendigkeit des Einfachen - eher dagegen zu sprechen scheint, ist es insgesamt wohl angebrachter, die eben zitierte Passage so zu deuten, daß Aristoteles dort nicht in inhaltlicher Absicht zwei weitere Notwendigkeiten neben den schon eingeführten einführt, sondern vielmehr eine modale Unterscheidung trifft und zwei Modi der Notwendigkeit spezifiziert, die alle schon eingeführten Notwendigkeiten, prima facie jedenfalls, annehmen können: Sie können, wo sie vorliegen, extrinsisch oder intrinsisch sein.

Daß alles, was form-notwendig ist, extrinsisch form-notwendig ist, ist zu verneinen (siehe dazu Abschnitt 6); Zweck- und EffizienzNotwendigkeit sind aber in der Tat wesenhaft generell extrinsisch. ${ }^{6}$

\footnotetext{
${ }^{6}$ Die Materie-Notwendigkeit hingegen läßt sich in keinem Fall extrinsisch oder intrinsisch nennen, weil Materie-Notwendigkeit stets grundlos ist: Alles, was materie-notwendig ist, hat weder in sich noch in einem anderen einen Grund für dieses Notwendigsein (das aus unserer Sicht, wie gesagt, eigentlich nicht als "Notwendigsein" anzusprechen ist). Darüber darf einen die Rede "Materie-
} 
Dasselbe gilt von der Beweisnotwendigkeit: Beweisnotwendig sind ja die bewiesenen Theoreme, und ihre Notwendigkeit qua bewiesene haben sie aufgrund der von ihnen zu unterscheidenden Axiome, aus denen sie logisch gefolgert sind; sie sind also stets extrinsisch beweisnotwendig (und intrinsische Beweisnotwendigkeit gibt es nicht). Allerdings zeigt sich hier ein Unterschied: Zwecke, die erfüllt werden, und Machtausübungen, die den außenliegenden Grund von Zweckbzw. Effizienz-Notwendigem bilden, hätten, so scheint es jedenfalls, meistenteils auch nicht gegeben sein können; die ersten Voraussetzungen eines Beweises, die also den außenliegenden Grund eines gewissen Beweisnotwendigen bilden, hätten hingegen, jedenfalls nach Aristoteles, nicht auch nicht gegeben sein können (siehe 1015 b 89). Bei extrinsischen Notwendigkeiten ist also zu unterscheiden, je nach kontingentem oder nicht-kontingentem außenliegenden Grund des jeweiligen Notwendigen. Weiter ist, wenn der Grund eines gewissen Notwendigseins von A nicht in ihm, sondern in etwas anderem, B, ist, zu unterscheiden, ob dies sich nur aus einem gewissen Blickwinkel der Ordnung der Erkenntnis, der Ordnung unseres Begründens so verhält, oder aber schlechthin objektiv so ist. Der Grund der Beweisnotwendigkeit von A mag bei gegebenem Axiomensystem im Axiom B liegen, A also extrinsisch beweisnotwendig sein; aber wechseln wir das Axiomensystem, so mag umgekehrt der Grund der Beweisnotwendigkeit von B im Axiom A liegen, dem nun als Axiom gar keine Beweisnotwendigkeit zukommt (sondern, nach Aristoteles, eine andere Notwendigkeit).

\section{Das weitere Schicksal der Notwendigkeit}

Was sich in der nachantiken Geistesgeschichte über längere Zeiträume hinweg insgesamt beobachten läßt, ist, daß die Materie-Notwendigkeit, die Zweck-Notwendigkeit und schließlich auch die Effizienz-Notwendigkeit als Quellpunkte von Notwendigkeitskonzeptionen immer mehr zugunsten der Form-Notwendigkeit an Bedeutung verlieren. Mit dem Beginn der Neuzeit und dem Abschied vom Mit-

notwendig ist, was durch die Materie notwendig ist" nicht hinwegtäuschen. 
telalter verloren sich die letzten Reste des bei allen Verwandlungen doch mit der anarchischen und vernunftabholden Materie-Notwendigkeit in Verbindung bleibenden Schicksalsglaubens. ${ }^{7}$ (Natürlich spreche ich hier immer nur bezogen auf die sogenannten "intellektuell fortschrittlichen Kreise", und auch diesbezüglich nur "im Durchschnitt".) Aber auch die Überzeugung von einer ultimativen Zweckbestimmung der Welt oder auch nur von einer ihr immanenten Zweckhaftigkeit geriet ins Wanken, und im weiteren Verlaufe der Neuzeit immer mehr. Heute ist die offizielle Meinung die, daß von einem nicht durch Menschen gesetzten Zweck der Welt oder auch nur von solchen Zwecken in der Welt nicht gesprochen werden kann. Es ist klar, daß damit, wenn es sich tatsächlich so verhält, die Zweck-Notwendigkeit jedenfalls aus der Natur verschwindet, denn es gibt ja dann keine Naturzwecke, auf die irgend Zweck-Notwendiges in der Natur bezogen werden könnte. Und in dem Maße, wie der Mensch gegenwärtig immer mehr als ein bloßer Teil einer Natur angesehen wird, die vollständig zweckfrei aufgefaßt wird, verschwindet die Zweck-Notwendigkeit auch aus dem menschlichen Bereich; denn auch durch Menschen gesetzte Zwecke gibt es im Sinne der Naturalisierung qua Physikalisierung des Menschen nicht in dem eigentlichen Sinn handlungsorientierender Prinzipien, die als solche in einem eigenen, von Aristoteles intendierten Sinn in der Bezugnahme auf sie, in ihrer Erfüllung etwas notwendig, nämlich zweckerforderlich machen, sondern höchstens im uneigentlichen Sinn kausal wirksamer humanpsychischer Ereignisse.

Wenn in diesem letzteren Satz gerade von der Kausalität die Rede war, die man im Sinne des aristotelischen Schemas gewöhnt ist, als "effiziente Kausalität" zu bezeichnen, so darf dies nicht zu der Meinung verführen, daß die Effizienz-Notwendigkeit heute noch eine

\footnotetext{
${ }^{7}$ Es ist eine der Ironien der Geistesgeschichte, daß der ontologische Zufall den Alten als tyche so vertraut - im 20. Jahrhundert wieder auf den intellektuellen Plan getreten ist. Freilich liegt es uns Heutigen fern, im Sinne Platons den ontologischen Zufall unter eine Notwendigkeit, eben die Materie-Notwendigkeit zu subsumieren. Deshalb kann von einer Renaissance der Materie-Notwendigkeit nicht recht gesprochen werden.
} 
Rolle spielt. Denn, was wir als "effiziente Kausalität" bezeichnen, ist als Folge der verheerenden Kritik David Humes vollständig von der Effizienz-Notwendigkeit dissoziiert worden. Wenn heute von "kausaler Notwendigkeit" gesprochen wird, dann ist damit eine Notwendigkeit gemeint, die auf der Form-Notwendigkeit, und nur auf ihr, gründet. Der Begriff der Effizienz-Notwendigkeit dagegen ist weitgehend obsolet geworden, worüber einen freilich die Übertragung von Konnotationen der Effizienz-Notwendigkeit auf die Form-Notwendigkeit in der modernen Rede von Kausalität, von angeblich "effizienter Kausalität", leicht hinwegtäuschen kann. Schon Malebranche (und schon vor ihm manch anderer, etwa Algazel) hat den Begriff der Effizienz-Notwendigkeit allein im Hinblick auf Gott für anwendbar gehalten, für anwendbar allein im Hinblick auf die Ausübung Seiner Macht: Was Gott will, geschieht unausbleiblich, ist effizienznotwendig; bei jedem anderen prima facie möglichen Agens besteht dieser Zusammenhang dagegen nicht. Die Anwendungskonzentration auf von Gott Gewolltes weist hier unmißverständlich auf die Unbegreifbarkeit des Begriffes der Effizienz-Notwendigkeit hin. Bei Gott kann man sich eben auf die Position zurückziehen, daß sein Wille per definitionem unwiderstehbar effektiv ist, und man gewinnt somit für Effizienz-Notwendigkeit wenigstens ein mögliches Anwendungsfeld (freilich ein höchst hypothetisches, denn erstens ist Gottes Existenz ungesichert, und zweitens ist, was er will, ungewiß). Aber einem Verständnis der Effizienz-Notwendigkeit ist man durch dieses Ausweichmanöver, die Verschiebung auf Gott, um keinen Deut näher gekommen.

\section{Form-Notwendigkeit und intrinsische Gesetze}

Von allen antiken Notwendigkeitsformen steht gegenwärtig allein die Form-Notwendigkeit in voller Blüte (wenn man so reden will), und sie tritt, so gut es geht, in die Rollen all der anderen, obsolet gewordenen Notwendigkeitsformen ein (einschließlich der Schicksalsnotwendigkeit). Es lohnt sich daher, dem besonderen Charakter der Form-Notwendigkeit nachzugehen. 
Form-Notwendigkeit ist, wie gesagt, die durch eine Form, eine gewisse Gestalt (in noch weiterem Sinn als das antike eidos und das mittelalterliche forma), nämlich die durch ein Gesetz gegebene Notwendigkeit. Dort, wo eine Form vorliegt oder, wie man auch sagt, erfüllt ist, exemplifiziert ist, ist, wenn diese Form ein Gesetz ist, entweder schon in jedem Erfüllungsfall dieser selbst, die jeweilige Erfüllung der Form, durch die Form erfordert und notwendig, mithin intrinsisch form-notwendig, oder aber die Form erfordert nur etwas von ihren (gleichwohl vorliegenden) Erfüllungen Verschiedenes, und es ist eben dieses andere (ein anderer Sachverhalt) durch sie notwendig, also, wenn es den Grund seiner Form-Notwendigkeit nur außerhalb von sich hat, extrinsisch form-notwendig. ${ }^{8}$ Beides kommt offenbar vor, und deshalb ist Form-Notwendigkeit weder schlechthin extrinsisch noch schlechthin intrinsisch.

Formen oder Gestalten (wie sie hier unter historischem, aber auch unter systematischem Gesichtspunkt aufgefaßt werden) sind nun gewisse Universalien. Beschränken wir uns der Vereinfachung halber darauf (was ja auch der Tradition entspricht), unter dem Wort "Form" ("Gestalt") diejenigen Universalien zu verstehen, die auf konkrete Individuen anwendbar und monadisch, d. h. nichtrelational, einstellig sind. ${ }^{9}$ Formen sind dann des weiteren entweder atemporal oder temporal, und die temporalen Formen wiederum sind

\footnotetext{
${ }^{8}$ Es ist intrinsisch form-notwendig, daß $F a$, gdw. es durch $F$ notwendig ist, daß $F a$; denn der Grund dafür, daß $F a$ form-notwendig ist, liegt ja genau dann in dem form-notwendigen Sachverhalt, daß Fa, selbst, wenn es durch $F$ notwendig ist, daß Fa. Entsprechend: Es ist extrinsisch form-notwendig, daß $G a$, gdw. es nicht durch $G$, sondern eine andere Form als $G$ notwendig ist, daß $G a$; denn der Grund dafür, daß $G a$ form-notwendig ist, liegt ja genau dann nicht in dem form-notwendigen Sachverhalt, daß Ga, selbst, sondern außerhalb, wenn es nicht durch $G$, sondern durch eine andere Form als $G$ notwendig ist, daß $G a$.

${ }^{9}$ Eine weitere mögliche Einschränkung wäre es, unter den auf konkrete Individuen anwendbaren monadischen Universalien nur solche als Formen zu betrachten, die keine konkreten Individuen als Konstituenten haben (sondern höchstens abstrakte Individuen, wie Zahlen, Zeitpunkte, etc.). Doch ist die Einschränkung des Formbegriffs auf reine Universalien wohl unter jedem Aspekt eine zuweitgehende. - Um der Sache voll gerecht $\mathrm{zu}$ werden, wäre es erforderlich, auch relationale Formen (insbesondere dyadische) zu betrachten; doch um der Komplexitätsreduktion willen soll dies hier unterbleiben.
} 
synchron oder diachron. Schließlich können Formen komplex oder einfach sein.

Betrachten wir einige Beispiele von Formen und ihr Verhalten im Hinblick auf die Form-Notwendigkeit:

(1) Mit sich selbst identisch zu sein, ist eine atemporale, einfache Form. Es ist form-notwendig, daß ich mit mir selbst identisch bin, und der Grund des Form-Notwendigseins dieses Form-Notwendigen liegt nicht in einem anderen, in etwas außerhalb von ihm: Daß ich die Form, mit sich selbst identisch zu sein, erfülle, ist ja durch diese Form selbst erfordert. Es ist demnach intrinsisch form-notwendig, daß ich identisch mit mir selbst bin. Mithin haben wir hier ein Beispiel für eine intrinsische Form-Notwendigkeit.

(2) $Z u t_{0}$ nicht $z u$ existieren oder $z u t_{0}$ ein Mensch zu sein, ist eine temporale, synchrone, komplexe Form. Es ist anscheinend notwendig, daß ich zu $t_{0}$ nicht existiere oder zu $t_{0}$ ein Mensch bin. Es ist aber nicht form-notwendig, daß ich zu $t_{0}$ nicht existiere oder zu $t_{0}$ ein Mensch bin. Denn es ist nicht durch eine Form notwendig, daß ich zu $t_{0}$ nicht existiere oder zu $t_{0}$ ein Mensch bin, sondern für das Notwendigsein dieses Notwendigen kommt es wesentlich auch auf mich an; ich aber bin eine Substanz und keine Form.

Allgemein können wir im Anschluß hieran festhalten:

\section{GEnERALISIERUngsPRINZIP DER ForM-NotwendigkeIT}

Wenn eine Erfüllung einer Form form-notwendig ist, dann ist jede Erfüllung der Form form-notwendig.

Denn die Form, die die eine Erfüllung der Form notwendig macht, muß auch jede andere Erfüllung der Form notwendig machen: Ist sie qua Form hinreichend für das Notwendigsein der einen Erfüllung, dann qua Form auch hinreichend für das Notwendigsein aller anderen Erfüllungen. Eine auf der Hand liegende Modifikation dieser Argumentation zeigt auch die Richtigkeit des folgenden: 


\section{GENERALISIERUNGSPRINZIP DER INTRINSISCHEN FORM-NOTWENDIGKEIT}

Wenn eine Erfüllung einer Form intrinsisch form-notwendig ist, dann ist jede Erfüllung der Form intrinsisch form-notwendig.

Nach dem Generalisierungsprinzip der Form-Notwendigkeit ergibt sich gemäß den obigen Feststellungen, daß keine Exemplifikation der Form, zu to nicht zu existieren oder zu to ein Mensch zu sein, form-notwendig ist. Gleichwohl sind manche dieser Exemplifikationen anscheinend notwendig (wie wir ebenfalls sahen), und deshalb sind auch abgesehen von Materie-, Effizienz- und Zweck-Notwendigkeit etc. manche Sachverhalte offenbar notwendig (nämlich individuelle Essenz-Sachverhalte), die nicht form-notwendig ist, wenn auch eine Form ganz wesentlich am Notwendigsein dieser Notwendigen beteiligt ist. ${ }^{10}$

(3) Nicht ganz blau oder nicht ganz gelb zu $t_{0}$ zu sein, ist abermals eine temporale, synchrone, komplexe Form. Im Einklang mit der oben erstgenannten, aber atemporalen und einfachen Form ist es intrinsisch form-notwendig, daß $x$ nicht ganz blau oder nicht ganz gelb zu $t_{0}$ ist, gleichgültig, welches $x$ - ob mich selbst oder ein anderes - man auch betrachtet. Mit sich selbst identisch zu sein, und ebenso, nicht ganz blau oder nicht ganz gelb zu $t_{0} z u$ sein,

${ }^{10}$ Die heute allgemein verwendete Darstellungsform der Notwendigkeit(en) von Sachverhalten ist es zu sagen, daß ein Sachverhalt genau dann (in dem und dem Sinne) notwendig ist, wenn er in allen (in dem und dem Sinne) möglichen Welten besteht. Es ist zu beachten, daß in allen (so und so) möglichen Welten zu bestehen, keine Notwendigkeitsform neben anderen Notwendigkeitsformen ist, sondern eben nur die heute allgemein verwendete (wegen ihrer Anschaulichkeit besonders attraktive, von vielen aber auch wegen ihrer augenscheinlich problematischen ontologischen Voraussetzungen strikt abgelehnte) Darstellungsform der Notwendigkeit bedeutet. Ob ich sage, $p$ ist durch die Form $F$ notwendig, oder aber, $p$ besteht durch die Form $F$ in allen gesetzlichen möglichen Welten, bleibt sich gleich. (Zur ontologischen Kontroverse um (bloße) Possibilia, auf die hier nicht näher eingegangen werden kann, sei verwiesen auf David Lewis' On the Plurality of Worlds und auf David Armstrongs A Combinatorial Theory of Possibility. Die beiden prominenten Autoren vertreten entgegengesetzte Standpunkte: Lewis' Realismus bzgl. Possibilia steht Armstrongs Fiktionalismus gegenüber.) 
sind universal exemplifizierte Formen, was die Form, zu to nicht zu existieren oder zu $t_{0}$ ein Mensch zu sein, ja nicht ist.

(4) Niemals geboren zu sein oder einmal zu sterben, ist eine temporale, diachrone, komplexe Form. Es ist der Fall, daß ich und jedes beliebige $x$ niemals geboren ist oder einmal stirbt; auch die Form, niemals geboren zu sein oder einmal zu sterben, ist mithin eine universal exemplifizierte Form. Ist es aber form-notwendig, daß ich, beispielsweise, niemals geboren bin oder einmal sterbe? Klar scheint, daß dies nicht intrinsisch form-notwendig ist: Die Erfüllung der fraglichen Form durch mich ist offenbar nicht durch diese Form selbst erfordert. Wenn es denn form-notwendig sein sollte, daß ich niemals geboren bin oder einmal sterbe, dann ist der Grund des Notwendigseins dieses Form-Notwendigen offenbar nicht die Form, niemals geboren zu sein oder einmal zu sterben, liegt der Grund dafür nicht im formalen Aspekt des form-notwendigen Sachverhalts selbst, sondern nur in einer Form außerhalb von ihm; d. h., dann wäre es extrinsisch form-notwendig, daß ich niemals geboren bin oder einmal sterbe. Die Frage ist: Welche außenliegende Form begründete dann dieses Exempel extrinsischer Form-Notwendigkeit?

Unter den drei universal exemplifizierten Formen, die wir bislang betrachtet haben - mit sich selbst identisch zu sein, nicht ganz blau oder nicht ganz gelb zu $t_{0}$ zu sein, niemals geboren zu sein oder einmal zu sterben - unterscheidet sich die dritte von den beiden übrigen dadurch, daß man sie bestenfalls als extrinsisches Gesetz bezeichnen würde, die beiden übrigen aber als intrinsische Gesetze. Diese sind intrinsische Gesetze eben deshalb, weil sie (a) universal erfüllt sind und (b) jede Erfüllung von ihnen durch sie, die Formen selbst, form-notwendig ist (d. h.: jede Erfüllung von ihnen intrinsisch form-notwendig ist). Man beachte hierbei, daß $(a)$ und (b) voneinander unabhängig sind:

Zum einen: Jede Erfüllung einer Form kann durch die Form selbst form-notwendig sein (intrinsisch form-notwendig sein), ohne daß doch die Form universal erfüllt ist. Das ist trivialerweise gegeben, wenn es überhaupt keine Erfüllung der Form gibt. Gibt es aber eine Erfüllung der Form, so ist es nun tatsächlich fraglich, ob (b) ohne 
(a) gegeben sein kann. Nehmen wir an, für die Form $F$ gelte $(b)$, aber ein $x$ erfülle $F$ nicht; zudem nehmen wir aber auch an, mindestens ein $y$ erfülle F. Diese Erfüllung ist dann durch die Form $F$ notwendig. Wie aber kann das sein, wenn andererseits $x F$ nicht erfüllt? Offenbar spielt dann die Differenz zwischen den konkreten Individuen $x$ und $y$ doch eine Rolle, und folglich kann die Erfüllung von $F$ durch $y$ nicht durch $F$ (gemeint im Sinne von: allein durch $F$ ) notwendig sein. - Diese Überlegungen stützen das folgende Prinzip:

Wenn eine Form erfüllt ist und jede Erfüllung von ihr intrinsisch form-notwendig ist, dann ist die Form universal erfüllt.

Wegen des GENERALISIERUNGSPRINZIPS DER INTRINSISCHEN Form-NotwendigkeIT ist es äquivalent mit:

UniversalisiERUNG BEI INTRINSISCHER Form-NotwENDIGKEIT Wenn eine Erfüllung einer Form intrinsisch form-notwendig ist, dann ist die Form universal erfüllt. ${ }^{11}$

Es gibt nun keinen Grund dagegen, sondern vielmehr jeden Grund dafür - insbesondere die entsprechende Adaptierung des eben angegebenen Arguments für UniverSALISIERUNG BEI INTRINSISCHER Form-Notwendigkeit -, auch das zugehörige allgemeinere Prinzip anzunehmen:

\section{Universalisierung BeI Form-NotwendigkeIT}

Wenn eine Erfüllung einer Form form-notwendig ist, dann ist die Form universal erfüllt. ${ }^{12}$

${ }^{11}$ Wie unschwer ersichtlich, ist die Konjunktion der Bedingungen $(a)$ und $(b)$ unter Voraussetzung des GENERALISIERUNGSPRINZIPS DER INTRINSISCHEN FORM-NOTWENDIGKEIT und der UNIVERSALISIERUNG BEI INTRINSISCHER FORM-NotwendigkeIT äquivalent mit "eine Erfüllung der (fraglichen) Form ist intrinsisch form-notwendig". (Man beachte, daß daraus, daß $F$ universal erfüllt ist, folgt, daß $F$ mindestens einmal erfüllt ist.)

${ }^{12}$ Nach dem GenerAlisierungSPRINZIP DER Form-Notwendigkeit ist die Universalisierung BeI FORM-NotwendigkeIT äquivalent mit: Wenn eine Form erfüllt ist und jede Erfüllung von ihr form-notwendig ist, dann ist die Form universal erfüllt. 
Dieses Prinzip steht im Hintergrund der insbesondere von Kant betonten Verknüpfung von Notwendigkeit mit Allgemeinheit. Ohne sich darüber des näheren Rechenschaft zu geben, meinte schon Kant mit "Notwendigkeit" - ganz im Sinne der in Abschnitt 5 skizzierten geistesgeschichtlichen Entwicklung seit der Antike - einzig und allein Form-Notwendigkeit (man könnte genausogut sagen: Gesetzes-Notwendigkeit), und für die Form-Notwendigkeit gilt eben das angegebene Universalisierungsprinzip, das man, äquivalent, auch in der folgenden Form schreiben kann:

Für alle Formen $F$ : für alle $x$ : Wenn es form-notwendig ist, daß $F x,{ }^{13}$ dann gilt für alle $y F y$.

Doch die Umkehrung hiervon:

I. Für alle Formen $F$ : für alle $x$ : Wenn für alle $y F y$ gilt, dann ist es form-notwendig, daß $F x$,

und auch die direkte Umkehrung der UNIVERSALISIERUNG BEI Form-NotwendigKeIT selbst:

II. Wenn eine Form universal erfüllt ist, dann ist (mindestens) eine Erfüllung der Form form-notwendig,

gelten nicht. Darum fallen Form-Notwendigkeit und Allgemeinheit nicht zusammen.

Denn zum anderen (in der Frage, ob die oben angegebenen Bedingungen $(a)$ und (b) voneinander unabhängig sind): Die Form, niemals geboren zu sein oder einmal zu sterben, ist zwar universal erfüllt (so glauben wir zumindest), aber es gibt Erfüllungen von ihr, z.B. die durch mich, die nicht durch sie selbst form-notwendig sind, sondern entweder überhaupt nicht form-notwendig sind, oder aber nur durch eine andere Form als sie, so daß der Grund des

${ }^{13}$ Der Ausdruck "für alle $x$ : Wenn es form-notwendig ist, daß $F x$ " kann in dem angegebenen Satz ersetzt werden durch: "Wenn für mindestens ein $x: F x$ und es form-notwendig ist, daß $F x$ " (das ist die Präzisierung von "eine Erfüllung von $F$ ist form-notwendig"), da er ersetzbar ist durch "Wenn für mindestens ein $x$ es form-notwendig ist, daß $F x$ ", und sich ja aus "es ist form-notwendig, daß $F x "$ " $F x$ " logisch ergibt. 
Form-Notwendigseins jener Erfüllungen dann nicht in ihnen selbst liegt, sondern in einem anderen. Es ergibt sich also zunächst einmal, daß, wie schon $(b)$ ohne $(a)$, auch $(a)$ ohne $(b)$ gegeben sein kann, und damit ist die Unabhängigkeit von $(a)$ und $(b)$ voneinander nun vollständig gezeigt.

Nehmen wir nun aber weiter an, die Erfüllung der genannten Form durch mich sei nicht nur nicht intrinsisch form-notwendig, sondern überhaupt nicht. Damit wäre $I$. widerlegt, und wegen des GENERALISIERUNGSPRINZIPS DER FORM-NotwEndigKeIT (seiner logischen Kontraposition) ergibt sich damit auch die Widerlegung von II. Nun könnte es freilich immerhin extrinsisch form-notwendig sein, daß ich niemals geboren bin oder einmal sterbe. Deshalb eignet sich zur Widerlegung von $I$. und II. eine andere Form besser, nämlich diese: niemals eine realexistente Kugel aus Gold von $1 \mathrm{~km}$ Durchmesser zu sein. Diese Form ist universal erfüllt (so glauben wir zumindest). Aber es ist weder durch diese Form noch durch irgendeine andere notwendig, daß die (mereologische) Summe allen Goldes niemals eine realexistente Goldkugel von $1 \mathrm{~km}$ Durchmesser ist.

\section{Form-Notwendigkeit, extrinsische Gesetze und Gesetze simpliciter}

Unter einem extrinsischen Gesetz ist eine Form zu verstehen, die $\left(a^{\prime}\right)$ universal erfüllt ist und bei der $\left(b^{\prime}\right)$ jede Erfüllung von ihr nicht durch sie selbst, sondern durch eine andere Form notwendig ist (d. h.: jede Erfüllung von ihr extrinsisch form-notwendig ist). $\left(b^{\prime}\right)$ läßt aber, so wie es dasteht (alle Erläuterungen außer Betracht gelassen), zwei Deutungen zu, die sich durch die Stellung des zweiten Quantors unterscheiden:

Die logisch schwächere Deutung von $\left(b^{\prime}\right): \forall p$ ( $p$ ist eine Erfüllung von $F \supset p$ ist nicht durch $F$ notwendig, aber es gibt ein $G$, so daß $p$ durch $G$ notwendig ist).

Die logisch stärkere Deutung von $\left(b^{\prime}\right)$ : Es gibt ein $G$ : $\forall p(p$ ist eine Erfüllung von $F \supset p$ ist nicht durch $F$ notwendig, aber $p$ ist 
durch $G$ notwendig).

Es ist aber nicht nötig, sich für eine der beiden Deutungen zu entscheiden, denn das folgende FundamentalprinziP DER GeneRALISIERUNG DER FORM-NOTWENDIGKEIT stellt in Anwesenheit von $\left(a^{\prime}\right)$ (aus dem folgt, daß es mindestens eine Erfüllung von $F$ gibt) Äquivalenz zwischen beiden Deutungen her:

\section{FundamentalprinziP DER GENERALISIERUnG DER FORM-NOTWENDIGKEIT}

Für alle $F$ und $G$ : Ist eine Erfüllung von $F$ durch $G$ notwendig, dann ist jede Erfüllung von $F$ durch $G$ notwendig. ${ }^{14}$

Man beachte, daß aus diesem FundAMENTALPRINZIP sowohl das GeneralisierungsprinziP Der Form-Notwendigkeit also auch das GeneralisierungsPrinzip DER INTRINSISCHEN FormNotwendigkeIT folgen (siehe hierzu die näheren Ausführungen im übernächsten Abschnitt bei P4, P5, P6). Die Rechtfertigung des FundamentalPRINZIPS vollzieht sich entlang der für die schwächeren Prinzipien vorgezeichneten Linien.

Die Form, niemals geboren zu sein oder einmal zu sterben, ist nun $\left(a^{\prime}\right)$ universal erfüllt, und außerdem ist jede Erfüllung von ihr nicht durch sie selbst notwendig. (Denn wäre eine Erfüllung von ihr durch sie selbst notwendig, so müßte es nach dem aufgestellten FundamentalprinziP DER GENERALISIERUng jede Erfüllung von ihr sein - was aber, wie schon festgestellt, nicht der Fall ist.) Bei der Form, niemals geboren zu sein oder einmal zu sterben, handelt es sich also nach dem oben Gesagen genau dann um ein extrinsisches

${ }^{14}$ Ang. dann, $\forall p$ ( $p$ ist eine Erfüllung von $F \supset p$ ist nicht durch $F$ notwendig, aber es gibt ein $G$, so daß $p$ durch $G$ notwendig ist). Wegen ( $\left.a^{\prime}\right)$ erhält man: $\exists p$ ( $p$ ist eine Erfüllung von $F$ ). Mithin wegen der Annahme: $\exists p$ ( $p$ ist eine Erfüllung von $F$, und es gibt ein $G$, so daß $p$ durch $G$ notwendig ist). Also mit dem Fundamentalprinzip: $\exists G \forall p$ ( $p$ ist eine Erfüllung von $F \supset p$ ist durch $G$ notwendig), also mit der Annahme: $\exists G \forall p$ ( $p$ ist eine Erfüllung von $F \supset p$ ist nicht durch $F$ notwendig, aber $p$ ist durch $G$ notwendig). - Der umgekehrte Weg (von der logisch stärkeren Deutung zur logisch schwächeren) ist eine reine Sache der Logik. 
Gesetz, wenn es eine (andere) Form gibt, durch die jede Erfüllung der erstgenannten Form notwendig ist. Nach dem FundamentalPRINZIP DER GENERALISIERUNG hat man eine solche Form schon dann, wenn man eine Form hat, durch die eine Erfüllung der Form, niemals geboren zu sein oder einmal zu sterben, notwendig ist. Gibt es eine solche Form?

Anstatt sich gleich um ein Beispiel zu bemühen, kann man zunächst allgemein erwägen, was bei einer Form $G$, durch die eine Erfüllung einer Form $F$ notwendig ist, gegeben sein muß. Das folgende liegt nahe:

\section{FundamentalPRINZIP DER Form-NECESSITATION}

Für alle $F$ und $G$ : Wenn eine Erfüllung von $F$ durch $G$ notwendig ist, dann gilt:

(i) $G$ ist erfüllt;

(ii) $G$ ist (wie $F$ ) universal erfüllt;

(iii) $G$ ist ein Gesetz;

(iv) $F$ ist intensionale Teileigenschaft von $G .{ }^{15}$

Durch die Form, niemals zu entstehen oder einmal zu vergehen, kurz $G^{*}$, scheint nun eine Erfüllung der Form, niemals geboren zu sein oder einmal zu sterben, kurz $F^{*}$, in der Tat notwendig zu sein, und $G^{*}$ und $F^{*}$ scheinen dabei allen vier angeführten Bedingungen zu genügen. Dieses Genügen ist, was (i) und (iv) angeht, sicher, ${ }^{16}$ was

\footnotetext{
${ }^{15} F$ ist intensionale Teileigenschaft von $G$ genau dann, wenn $\forall x(G x \supset F x)$ analytisch wahr ist. So ist z.B. die Form, ein Mann zu sein, intensionale Teileigenschaft der Form, ein Junggeselle zu sein.

${ }^{16} \mathrm{Daß}$ die Form $F^{*}$, niemals geboren zu sein oder einmal zu sterben, eine intensionale Teileigenschaft der Form $G^{*}$, niemals zu entstehen oder einmal zu verge$h e n$, ist, sieht man ein, indem man die analytische Wahrheit von $\forall x\left(G^{*} x \supset F^{*} x\right)$ herleitet (vgl. die vorausgehende Fußnote). Dabei ist von folgenden analytischen Prinzipien Gebrauch zu machen: (1) Was einmal geboren ist, entsteht einmal. (2) Was einmal geboren ist, ist ein Lebewesen. (3) Was einmal vergeht und ein Lebewesen ist, stirbt einmal.
} 
(ii) angeht, höchst wahrscheinlich. Was aber (iii) angeht, zeigt sich ein gewisses Problem, auf das wir sogleich zurückkommen werden.

(iv) jedenfalls macht deutlich, wie es zugeht, daß durch $G^{*}$ eine Erfüllung von $F^{*}$ notwendig ist, wenn denn eine Erfüllung von $F^{*}$ durch $G^{*}$ notwendig ist: Wenn $G^{*}$ ein Gesetz ist ((iii) genügt, und folglich auch (ii) und (i)), dann überträgt die Relation Intensionale Teileigenschaft die mit diesem Gesetz verbundene Notwendigkeit auf eine, und mithin jede, Erfüllung der Teileigenschaft $F^{*}$ von $G^{*}$; außerdem wird durch die Relation Intensionale Teileigenschaft die Universalität der Erfüllung, die mit dem Gesetzesstatus ebenfalls verbunden ist, von $G^{*}$ auf $F^{*}$ übertragen.

Ist aber nun, niemals zu entstehen oder einmal zu vergehen, ein Gesetz? Prima facie sind wir schnell bereit, dies zu bejahen. In der Tat kann man sich fragen, was denn - abgesehen von mathematischen und logischen Gesetzen - überhaupt ein Gesetz sein sollte, wenn nicht, niemals zu entstehen oder einmal zu vergehen, ein Gesetz ist. Dabei setzen wir aber einen Gesetzesbegriff voraus, wonach eine Form einfach genau dann ein Gesetz ist, wenn sie notwendigerweise universal erfüllt ist, ${ }^{17}$ und wir lassen uns damit auf einen höchst ungeklärten Notwendigkeitsbegriff ein, demzufolge auf ein Urteil, dessen Begründung nicht recht ersichtlich ist.

Im Sinne der obigen Ausführungen zu extrinsischen und intrinsischen Gesetzen wäre vielmehr unter einem Gesetz (simpliciter) eine Form zu verstehen, die $\left(a^{\prime \prime}\right)$ universal erfüllt ist und bei der $\left(b^{\prime \prime}\right)$ jede ihrer Erfüllungen durch eine Form notwendig ist (d. h.: jede ihrer Erfüllungen form-notwendig ist). ( $\left.b^{\prime \prime}\right)$ läßt an sich die beiden logisch verschiedenen Deutungen $\left(b^{\prime \prime}{ }_{1}\right)$ " $\forall p(p$ ist eine Erfüllung von $F \supset \exists H(p$ ist durch $H$ notwendig $))$ " und $\left(b^{\prime \prime}{ }_{2}\right)$ " $\exists H \forall p$ ( $p$ ist eine Erfüllung von $F \supset p$ ist durch H notwendig)" zu; aber die Gegenwart von $\left(a^{\prime \prime}\right)$ und das Fundamentalprinzip DER GEneraliSIERUNG DER ForM-NotwendigkeIT sorgen für die Äquivalenz der beiden Deutungen.

\footnotetext{
${ }^{17}$ Wir schließen nämlich so: Niemals zu entstehen oder einmal zu vergehen ist ein Gesetz genau deshalb, weil es notwendigerweise so ist, daß alles, was einmal entsteht, einmal vergeht.
} 
Soll demnach durch die Form $G^{*}$ eine Erfüllung der Form $F^{*}$ notwendig sein, was $F^{*}$ als Gesetz etablieren würde, so muß $G^{*}$ selbst ein Gesetz sein, d. h. $G^{*}$ muß universal erfüllt sein und es muß eine Form $H$ geben, durch die jede Erfüllung von $G^{*}$ notwendig ist. Die universale Erfüllung von $G^{*}$ ist, wie gesagt, plausibel, aber $G^{*}$ ist offenbar nicht selbst die Form $H$, durch die jede Erfüllung von $G^{*}$ notwendig ist. Um den Gesetzes-Charakter von $G^{*}$ zu etablieren, findet man sich also weiterverwiesen auf eine weitere Form $H$. Selbst wenn man nun wüßte, welche Form $H$ ist, bei ihr, um den Gesetzes-Charakter von $G^{*}$ zu etablieren, stellt sich dieselbe Frage wie bei $G^{*}$, um den Gesetzes-Charakter von $F^{*}$ zu etablieren: Ist sie ein Gesetz? Es droht also die Gefahr eines infiniten Regresses im Bemühen um die Erkenntnis des Gesetzes-Charakters von Formen, die keine intrinsischen Gesetze sind.

\section{Gesetzes-Regresse und die Problematik von Naturgesetzen und der Form-Notwendigkeit selbst}

Wir sagen, daß eine Form $F$ durch eine Form $G$ Gesetz ist, genau dann, wenn es eine Erfüllung von $F$ gibt, die durch $G$ notwendig ist. Wie man leicht einsieht, ist dann $F$ ein intrinsisches Gesetz genau dann, wenn $F$ durch $F$ Gesetz ist, und ein extrinsisches Gesetz, wenn $F$ nicht durch $F$ Gesetz ist, es aber ein $G$ gibt, durch das $F$ Gesetz ist (siehe im nächsten Abschnitt die Beweise von P10 und P11). Außerdem läßt sich leicht beweisen, daß eine Form genau dann ein Gesetz ist, wenn sie durch eine Form Gesetz ist, die selbst Gesetz ist (siehe im nächsten Abschnitt den Beweis von P12). Und des weiteren: Jedes Gesetz ist entweder extrinsisch oder intrinsisch (siehe im nächsten Abschnitt den Beweis von P13). Schließlich gilt auch - unter der sehr plausiblen Voraussetzung, daß ein Sachverhalt, der durch eine Form notwendig ist, auch notwendig ist durch jede Form, die jene Form als intensionale Teileigenschaft hat (PRINZIP DER Form-Verstärkung) - die Aussage: Das Relationsprädikat " $X$ ist durch $Y$ Gesetz" ist transitiv (siehe im nächsten Abschnitt den Beweis von P15 unter Verwendung von P14, dem PRINZIP DER 
FORM-VERSTÄRKUNG).

Unter einer (nomologischen) Fundierungskette einer Form ist eine Folge von Formen zu verstehen, die ein erstes Glied hat (und gegebenenfalls auch nur aus diesem Glied besteht), die genau dann bei einem Glied aufhört, wenn das Glied durch sich selbst Gesetz ist, und in der jedes Glied, wenn es einen unmittelbaren Nachfolger hat, durch diesen Gesetz ist. Eine Fundierungskette einer Form besteht also aus Gesetzen, wobei diejenige Form, deren Fundierungskette die Kette ist, das erste Glied in ihr ist.

Es ist ersichtlich, daß eine Form genau dann ein Gesetz ist, wenn sie eine Fundierungskette hat. Weiter ist ersichtlich: Das letzte Glied einer Fundierungskette, so es ein solches gibt, ist stets ein intrinsisches Gesetz, und alle ihre Glieder zwischen dem ersten und dem letzten sind extrinsische Gesetze; wenn die Kette mehr als ein Glied hat, so ist außerdem ihr erstes Glied stets ein extrinsisches Gesetz. Eine Fundierungskette einer Form kann zudem keine Wiederholungen enthalten (ein Glied an einer Stelle in ihr kann nicht mit einem Glied an anderer Stelle in ihr identisch sein): Denn nehmen wir an, in einer solchen Kette tritt neu $F$ auf und später in ihr noch einmal $F$, entweder unmittelbar nach dem ersten Auftreten von $F$ oder nach einigen Zwischengliedern; im ersten Fall wäre $F$ durch sich selbst Gesetz, und im zweiten Fall ebenfalls (wegen der Transitivität des Fundierungsprädikats " $X$ ist durch $Y$ Gesetz"). Dann hätte die Kette aber, als Fundierungskette einer Form, schon beim ersten Auftreten von $F$ abbrechen müssen (laut ihrer Definition) im Widerspruch zur Annahme.

Prima facie können Fundierungsketten von Gesetzen eingliedrig sein, oder mehrgliedrig endlich, oder unendlich. Alle Fundierungsketten von intrinsischen Gesetzen sind eingliedrig, und alle eingliedrigen Fundierungsketten sind solche von intrinsischen Gesetzen. Alle Fundierungsketten von extrinsischen Gesetzen sind mehrgliedrig, und alle mehrgliedrigen Fundierungsketten sind solche von extrinsischen Gesetzen. Die mehrgliedrig endlichen Fundierungsketten, die mehr als zwei Glieder haben, brauchen nicht weiter berücksichtigt werden, denn es läßt sich leicht zeigen, daß wenn ein Gesetz eine 
mehrgliedrig endliche Fundierungskette hat, daß es dann auch eine zweigliedrige Fundierungskette hat (wegen der Transitivität der Fundierungsrelation). Des weiteren gilt: Jede Fundierungskette von $F$ ist genau dann unendlich, wenn es kein intrinsisches Gesetz gibt, durch das $F$ Gesetz ist. ${ }^{18}$

Gibt es nun Gesetze, so daß es kein intrinsisches Gesetz gibt, durch das sie Gesetze sind? Es ist gut möglich, daß niemals geboren $z u$ sein oder einmal zu sterben ein solches Gesetz ist. Ebenso ist es gut möglich, daß niemals zu entstehen oder einmal zu vergehen ein solches Gesetz ist. Das Problem ist aber, daß sich Gesetze mit ausschließlich unendlichen Fundierungsketten (Gesetze, die nicht durch ein intrinsisches Gesetz Gesetz sind) nicht als solche erkennen lassen: Es ist bei einem Gesetz $F$ ohne endliche Fundierungskette unerkennbar, daß $F$ ein Gesetz ist; denn es ist ohne endliche Fundierungskette von $F$ weder evident, daß $F$ ein Gesetz ist, noch gibt es eine korrekte Begründung dafür, daß $F$ ein Gesetz ist. Eine korrekte Begründung dafür, daß $F$ ein Gesetz ist, muß nämlich durch die Angabe eines anderen Gesetzes $H$ erfolgen, durch das $F$ Gesetz ist und dessen Gesetzesstatus leichter einzusehen ist als der von $F$. Gäbe es ein intrinsisches anderes Gesetz $H$, durch das $F$ Gesetz ist, so gäbe es wohl auch eine solche Begründung - sonst aber nicht: Der bloße Verweis auf ein (putatives) extrinsisches anderes Gesetz $H$, durch das $F$ Gesetz ist, genügt nicht, da der Gesetzesstatus von $H$ nicht leichter einzusehen ist als der von $F$; denn wie $F$ ist $H$ ja höchstens durch ein anderes Gesetz Gesetz. Man kann auch wie folgt argumentieren: Die Erkenntnis, daß $F$ ein Gesetz ist, erfordert die Erkenntnis einer endlichen Fundierungskette von $F$. Es gibt aber keine endliche Fundierungskette von $F$ (gemäß Voraussetzung). Deshalb gibt es davon, daß $F$ ein Gesetz ist, keine Erkenntnis.

\footnotetext{
${ }^{18}$ Wenn es ein intrinsisches Gesetz $G$ gibt, durch das $F$ Gesetz ist, dann gibt es eine endliche Fundierungskette von $F$, nämlich, wenn $F$ ein intrinsisches Gesetz ist, einfach $\langle F\rangle$, wenn aber $F$ ein extrinsisches Gesetz ist, $\langle F, G\rangle$. Umgekehrt: Wenn es eine endliche Fundierungskette von $F$ gibt, dann gibt es ein intrinsisches Gesetz, durch das $F$ Gesetz ist; $F$ ist nämlich insbesondere Gesetz durch das letzte Glied der Kette, und dieses ist ein intrinsisches Gesetz.
} 
Es sieht nun ganz so aus, als wären alle extrinsischen Gesetze Gesetze mit ausschließlich unendlichen Fundierungsketten. Denn nehmen wir an, $F$ ist ein extrinsisches Gesetz und es gäbe ein intrinsisches Gesetz $G$, durch das $F$ Gesetz ist. Nach dem FundAMENTALPRINZIP DER FORM-NECESSITATION ist also $F$ intensionale Teileigenschaft von G. Nun sieht es aber so aus, als wären die intrinsischen Gesetze genau die logischen Formen (d. h. hier: die logischen Eigenschaften konkreter Individuen) ${ }^{19}$ Jede intensionale Teileigenschaft einer logischen Form (jede durch sie analytisch implizierte Form) ist aber selbst eine logische Form. Mithin ist $F$ wie $G$ eine logische Form, und folglich ein intrinsisches Gesetz, und also gar kein extrinsisches Gesetz im Widerspruch zur Annahme.

Diese Überlegungen sind deshalb bedeutsam, weil sie, wenn sie richtig sind, für die Konzeption von sogenannten Naturgesetzen schwerwiegende Konsequenzen haben. Die einfachsten Naturgesetze wird man nämlich mit gewissen derjenigen Universalien gleichsetzen können, die wir hier als "Formen" bezeichnet haben; ${ }^{20}$ ausnahmslos handelt es sich dann bei ihnen sicherlich nicht um logische Formen, also auch nicht um intrinsische Gesetze. Wenn sie also überhaupt Gesetze im definierten Sinn sind, dann müssen sie extrinsische Gesetze sein. Aber nach dem eben Gesagten haben sie dann keine endliche Fundierungskette und sind folglich nicht als Gesetze erkennbar.

Gegen diese skeptische Argumentation zum Thema Naturgesetze, wonach gerade die einfachsten sogenannten Naturgesetze entweder gar keine Gesetze sind, oder aber zwar Gesetze sind, aber als solche unerkennbar, ließe sich einwenden, daß sie einen idiosynkratischen und irrelevanten Gesetzesbegriff voraussetze; gemäß dem relevanten

${ }^{19}$ Daran, daß die logischen Formen intrinsische Gesetze sind, kann kein Zweifel sein. Als fraglich könnte allein gelten, ob alle intrinsischen Gesetze logische Formen sind.

${ }^{20}$ Naturgesetze als gewisse Universalien aufzufassen statt als gewisse Sachverhalte oder Sätze, paßt am besten zu der üblichen, weitverbreiteten Einordnung von ihnen als Regularitäten in der Natur; weder Sachverhalte oder Sätze sind ja Regularitäten. - Man beachte, daß zwar alle Naturgesetze Regularitäten in der Natur sind, aber nicht alle Regularitäten in der Natur Naturgesetze. 
Gesetzesbegriff gebe es selbstverständlich Beispiele für erkennbare Naturgesetze. Hierzu ist zu sagen, daß erstens nicht ersichtlich ist, warum der hier verwendete, explizit definierte Gesetzesbegriff irrelevant sein soll, und zweitens, daß bei den angeblichen Beispielen von erkennbaren Naturgesetzen deutlich wird, daß das einzige einigermaßen gesicherte objektive Kriterium, das bei der Auszeichnung einer Form als Naturgesetz zur Anwendung kommt, die universale Erfüllung ist; der kriteriale Rest (er läßt sich unter dem Titel "große naturwissenschaftliche Bedeutung" zusammenfassen) hat vor allem mit uns und unserer Erkenntnis zu tun, hat also keinen objektiven Charakter. ${ }^{21}$ Natürlich halten wir aus diesen oder jenen Gründen gewisse universale Formen für Naturgesetze; aber einen von uns erkannten hinreichenden objektiven Grund dafür können wir nicht nennen: Die Gründe, die wir nennen können, sind entweder von uns erkannt, aber nicht objektiv, oder aber zwar objektiv, aber nicht von uns erkannt, sondern nur rein spekulativ unterstellt, also gar keine Gründe, sondern metaphysische Hypothesen. ${ }^{22}$

Auch die Form-Notwendigkeit, jedenfalls soweit sie extrinsisch sein soll, erweist sich also letztlich als ein problematischer Begriff - wie vor ihr in der Geistesgeschichte seit der Antike schon die Zweck-Notwendigkeit und die Effizienz-Notwendigkeit. Es ist hinreichend klar, was es bedeutet, daß eine Erfüllung der Form, nicht ganz blau oder nicht ganz gelb zu $t_{0}$ zu sein, - etwa deren Erfüllung durch diesen Ball dort - durch diese Form selbst notwendig ist; es ist aber nicht hinreichend klar, was es bedeutet, daß eine Erfüllung

${ }^{21}$ Immer wieder wird als Gesetzeskriterium die Fähigkeit bemüht, kontrafaktische Konditionale zu stützen. Z.B.: Wenn neben der universalen Erfüllung der Form non- $F$ oder $G$ auch für beliebige $x$, die nicht $F$ und nicht $G$ erfüllen, gilt: wäre $x$ ein $F$, so wäre es ein $G$, so sei das ein (hinreichendes?) Zeichen dafür, daß non- $F$ oder $G$ ein Gesetz ist. Das Problem hierbei ist, daß niemand von der Wahrheit von "wäre $x$ ein $\mathrm{F}$, so wäre es ein G" für beliebige $x$, die nicht $F$ und nicht $G$ sind, überzeugt ist, wenn er nicht schon davon überzeugt ist, daß non- $F$ oder $G$ ein Gesetz ist; letztere Überzeugung muß also ihre Begründung, wenn sie eine hat, in etwas anderem als in jener ersteren Überzeugung finden.

${ }^{22}$ Letzteres gilt insbesondere für die einflußreiche Naturgesetzkonzeption von David Armstrong in What Is a Law of Nature? Zur Problematik des Naturgesetzbegriffes vgl. Meixner, Ereignis und Substanz. 
der Form, niemals geboren zu sein oder einmal zu sterben, durch irgendeine Form notwendig ist. ${ }^{23}$ Nicht nur bei dem Versuch, dies als wahr zu begründen, sondern auch, in eins damit, bei dem Versuch, zur Klarheit darüber zu kommen, was es denn besagt, gerät man in einen immer weiterschreitenden Regreß, in dem sich bei jedem Schritt dieselbe Unklarheit wiederholt. Sollte niemals geboren zu sein oder einmal zu sterben in der Tat ein Gesetz sein, d. h. in der Tat eine Erfüllung dieser Form durch irgendeine Form notwendig sein, so sieht es ganz danach aus, wie wir gesehen haben, als bliebe gerade deshalb, nicht nur ad infinitum die Erkennbarkeit dieser (unterstellten) Tatsache aufgeschoben, sondern auch ad infinitum die vollständige Beantwortung der Frage, was das eigentlich besagt: "eine Erfüllung von niemals geboren zu sein oder einmal zu sterben ist durch eine Form notwendig", "niemals geboren zu sein oder einmal zu sterben ist ein Gesetz". Denn jede Fundierungskette von niemals geboren zu sein oder einmal zu sterben wäre - so sieht es ja aus - dann, wenn diese Form Gesetz ist, unendlich. Ja, auch wenn wir ex impossibile eine ganze Fundierungskette jener Form als Gesetz durchlaufen könnten, so wären wir einer vollständigen Beantwortung jener Frage um keinen Deut näher.

Der einzige Ausweg aus dieser mißlichen Lage wäre, daß es doch, entgegen dem Anschein, intrinsische Gesetze gibt, die keine logischen Formen sind. Dann könnte, das Gesetzsein von, niemals geboren zu sein oder einmal zu sterben, mittels der Relation $F$ ist durch $G$ Gesetz (das ist keine andere Relation als die Relation $F$ ist intensionale Teileigenschaft von $G$, und $G$ ist Gesetz; siehe im nächsten Abschnitt P16 und P17) aufruhen auf dem wohlverstandenen Gesetzsein eines solchen intrinsischen Gesetzes (freilich ist Intrinsität noch keine Garantie für die leichte epistemische Zugänglichkeit eines Gesetzes, wofür schon manche logische Formen Beispiele bieten). Doch sieht es eben nicht im mindesten danach aus, daß es intrinsische Gesetze gibt, die keine logischen Formen sind.

\footnotetext{
${ }^{23}$ Noch viel unklarer müßte es sein, wenn die Erfüllung der Form durch irgendetwas anderes als eine Form notwendig sein soll.
} 


\section{Eine Semi-Formalisierung der Theorie der Form-Notwendigkeit für monadische Formen von konkreten Individuen (Appendix)}

Wir verwenden eine umgangssprachlich durchsetzte prädikatenlogische Sprache mit Alloperator " $\forall$ " und Existenzoperator " $\exists$ " und mindestens drei Variablentypen: $p, q, r, p^{\prime}, q^{\prime}, \ldots ; x, y, z, x^{\prime}$, $y^{\prime}, \ldots ; F, G, H, F^{\prime}, G^{\prime}, \ldots$ Den eben angeführten Variablentypen korrespondieren im Grundbereich der Sprache drei Sorten von Entitäten: Sachverhalte, konkrete Individuen, monadische Formen (Eigenschaften) konkreter Individuen. Von Bedeutung ist außerdem, daß die verwendete Sprache den Nominalisator "daß” enthält, der aus einem beliebigen Satz $A$ den singulären, durch Sachverhaltsvariablen ersetzbaren Term "[der Sachverhalt,] daß $A$ " bildet, wobei stets das folgende Schema erfüllt ist: Daß A, ist der Fall $\equiv$ A. ("三" und " $\supset$ " stehen für die sogenannte materiale Äquivalenz bzw. sogenannte materiale Implikation; sie binden schwächer als alle anderen Satzoperatoren.)

\section{Definitionen:}

D1 $p$ ist ein Sachverhalt $:=\exists q(p=q)$.

D2 $x$ ist ein konkretes Individuum $:=\exists y(x=y)$.

D3 $F$ ist eine (monadische) Form / Gestalt (konkreter Individuen) $:=\exists G(F=G)$.

D4 $p$ ist form-notwendig $:=\exists H$ ( $p$ ist durch $H$ notwendig).

D5 $p$ ist intrinsisch form-notwendig := $p$ ist form-notwendig, und der Grund des Form-Notwendigseins von $p$ ist in $p$.

D6 $p$ ist extrinsisch form-notwendig := $p$ ist form-notwendig, und der Grund der Form-Notwendigseins von $p$ ist nicht in $p$.

D7 $p$ ist eine Erfüllung von $F:=\exists y(F y$ und $p=\operatorname{daß} F y)$. 
D8 $F$ ist erfüllt / exemplifiziert $:=\exists y F y$. [Äquivalent: $\exists p$ ( $p$ ist eine Erfüllung von $F$ ).]

D9 $F$ ist universal erfüllt / exemplifiziert $:=\forall y F y$.

D10 $F$ ist aktual $:=F$ ist erfüllt.

D11 $F$ ist ein intrinsisches Gesetz $:=\forall y F y$ und $\forall p$ ( $p$ ist eine Erfüllung von $F \supset p$ ist durch $F$ notwendig).

D12 $F$ ist ein extrinsisches Gesetz $:=\forall y F y$ und $\forall p$ ( $p$ ist eine Erfüllung von $F \supset p$ ist nicht durch $F$ notwendig, aber es gibt ein $G$, so daß $p$ durch $G$ notwendig ist).

D13 $F$ ist ein Gesetz $:=\forall y F y$ und $\forall p$ ( $p$ ist eine Erfüllung von $F \supset$ es gibt ein $G$, so daß $p$ durch $G$ notwendig ist).

D14 $F$ ist durch $G$ Gesetz $:=\exists p$ ( $p$ ist eine Erfüllung von $F$, und $p$ ist durch $G$ notwendig).

\section{Prinzipien und Theoreme:}

P0 $\forall F \forall p(p$ ist durch $F$ notwendig $\supset p$ ist der Fall). [Daß P0 gilt, liegt auf der Hand: Wenn $p$ durch $F$ notwendig ist, dann ist $p$ durch $F$ der Fall, also auch schlicht der Fall. Eine interessante Frage ist es, ob man einen Unterschied machen sollte zwischen durch $F$ notwendig zu sein und durch $F$ der Fall zu sein. Ich würde die Frage verneinen: Durch $F$ der Fall zu sein, d. h., allein aufgrund der Form $F$ der Fall zu sein, scheint mir nichts anderes zu sein als, durch $F$ notwendig zu sein.]

P1 $\forall F \forall x$ : Daß $F x$, ist eine Erfüllung von $F \equiv F x$. [Folgt aus D7, wenn aus "daß $F x=$ daß $F y$ " folgt: " $x=y$ ". Es kommt ganz auf den Sachverhaltsbegriff an, ob diese letztere Folgerungsbeziehung gilt.]

P2 $\forall F \forall x$ : Daß $F x$, ist intrinsisch form-notwendig $\equiv \operatorname{da} \beta F x$, ist durch $F$ notwendig. [Ergibt sich aus D4 und D5, wenn "der 
Grund des Form-Notwendigseins des Sachverhalts, daß $F x$, ist in dem Sachverhalt, daß $F x$ " äquivalent ist mit "daß $F x$, ist durch $F$ notwendig". ${ }^{24}$ ]

P3 $\forall F \forall x$ : Daß $F x$, ist extrinsisch form-notwendig $\equiv$ daß $F x$, ist nicht durch $F$ notwendig, aber es gibt ein $G$, durch welches, daß $F x$, notwendig ist. [Ergibt sich mit D4 und D6, wenn "der Grund des Form-Notwendigseins des Sachverhalts, daß $F x$, ist nicht in dem Sachverhalt, daß $F x$ " äquivalent ist mit "daß $F x$, ist nicht durch $F$ notwendig".]

P4 $\forall F \forall G$ : $\exists p$ ( $p$ ist eine Erfüllung von $F$ und $p$ ist durch $G$ notwendig) $\supset \forall p$ ( $p$ ist eine Erfüllung von $F \supset p$ ist durch $G$ notwendig). [FundamentalprinziP DER Generalisierung DER Form-Notwendigkeit.]

P5 $\forall F: \exists p$ ( $p$ ist eine Erfüllung von $F$ und $p$ ist form-notwendig) $\supset \forall p$ ( $p$ ist eine Erfüllung von $F \supset p$ ist form-notwendig). [(Gewöhnliches) GeneralisierungsprinziP Der Form-NotWENDIGKEIT; P5 folgt aus P4 mit D4.]

$\ddot{A}$ quivalent: $\forall F: \exists x \exists G$ (daß $F x$, ist durch $G$ notwendig) $\supset \forall x(F x \supset$ $\exists G$ (daß $F x$, ist durch $G$ notwendig)).

P6 $\forall F: \exists p$ ( $p$ ist eine Erfüllung von $F$, und $p$ ist intrinsisch form-notwendig) $\supset \forall p$ ( $p$ ist eine Erfüllung von $F \supset p$ ist intrinsisch form-notwendig). [GENERALISIERUNGSPRINZIP DER INTRINSischen Form-Notwendigkeit; P6 folgt aus P4. ${ }^{25}$ ]

\footnotetext{
${ }^{24} \mathrm{Daß}$ sich die erste Aussage aus der zweiten ergibt, ist klar. Etwas weniger klar ist, ob sich auch die zweite Aussage aus der ersten ergibt. Doch scheint man mit der Annahme, daß es sich so verhält, ganz in seinem Recht zu sein.

${ }^{25}$ Ang., $\exists p$ ( $p$ ist eine Erfüllung von $F$, und $p$ ist intrinsisch form-notwendig), also mit D7: $\exists p \exists x$ ( $F x$ und $p=\operatorname{da} \beta F x$ und $p$ ist intrinsisch form-notwendig), also: $\exists p \exists x$ ( $F x$ und $p=$ daß $F x$ und, daß $F x$, ist intrinsisch form-notwendig), also mit P2: $\exists p \exists x$ ( $F x$ und $p=$ da $F x$, und, daß $F x$, ist durch $F$ notwendig), also: $\exists p \exists x$ ( $F x$ und $p=\operatorname{da} \beta F x$, und $p$ ist durch $F$ notwendig), also mit D7: $\exists p$ ( $p$ ist eine Erfüllung von $F$, und $p$ ist durch $F$ notwendig), also mit P4: $\forall p(p$ ist eine Erfüllung von $F \supset p$ ist durch $F$ notwendig), also mit D7: $\forall p \forall x(F x$ und $p=\operatorname{da} \beta F x \supset p$ ist durch $F$ notwendig), also: $\forall p \forall x$ (Fx und $p=$ daß $F x \supset$
} 
Äquivalent: $\forall F: \exists x$ (daß $F x$, ist durch $F$ notwendig) $\supset \forall x(F x \supset$ daß $F x$, ist durch $F$ notwendig) ${ }^{26}$

P7 $\forall F \forall G$ : $\exists p$ ( $p$ ist eine Erfüllung von $F$, und $p$ ist durch $G$ notwendig) $\supset G$ ist ein Gesetz, und $F$ ist eine intensionale Teileigenschaft von G. [FundamentalprinzIP DER FormNecessitation.]

P8 $\forall F: \exists p$ ( $p$ ist eine Erfüllung von $F$, und $p$ ist form-notwendig) $\supset \forall x F x$. [Universalisierung Bei Form-Notwendigkeit; P8 folgt aus P7. $\left.{ }^{27}\right]$

Äquivalent: $\forall F: \exists x \exists G$ (daß $F x$, ist durch $G$ notwendig) $\supset \forall x F x$.

P9 $\forall F: \exists p$ ( $p$ ist eine Erfüllung von $F$, und $p$ ist intrinsisch formnotwendig) つ $\forall x F x$. [UNIVERSALISIERUNG BEI INTRINSISCHER Form-Notwendigkeit. P9 folgt aus P8, da was intrinsisch formnotwendig ist, auch form-notwendig ist.]

Äquivalent: $\forall F: \exists x$ (daß $F x$, ist durch $F$ notwendig) $\supset \forall x F x$.

P10 $\forall F: F$ ist ein intrinsisches Gesetz $\equiv F$ ist durch $F$ Gesetz.

daß $F x$, ist durch $F$ notwendig $)$, also mit $\mathrm{P} 2: \forall p \forall x(F x$ und $p=$ daß $F x \supset$ daß $F x$, ist intrinsisch form-notwendig), also: $\forall p(\exists x(F x$ und $p=\operatorname{daß} F x) \supset p$ ist intrinsisch form-notwendig), also mit D7: $\forall p$ ( $p$ ist eine Erfüllung von $F \supset p$ ist intrinsisch form-notwendig).

${ }^{26} \exists p$ ( $p$ ist eine Erfüllung von $F$, und $p$ ist intrinsisch form-notwendig), d. h. nach D7: $\exists p(\exists x(F x$ und $p=$ daß $F x)$ und $p$ ist intrinsisch form-notwendig $)$, d. h.: $\exists x(F x$ und $\exists p$ ( $p=\operatorname{da} \beta F x$, und $p$ ist intrinsisch form-notwendig) $)$, d. h.: $\exists x(F x$ und, daß $F x$, ist intrinsisch form-notwendig), d. h. nach P2: $\exists x(F x$ und, daß $F x$, ist durch $F$ notwendig), d. h. nach P0: $\exists x(d a \beta F x$, ist durch $F$ notwendig). (Äquivalenz der Vorderglieder.)

$\forall p$ ( $p$ ist eine Erfüllung von $F \supset p$ ist intrinsisch form-notwendig), d. h. nach D7: $\forall p(\exists x(F x$ und $p=\operatorname{da} \beta F x) \supset p$ ist intrinsisch form-notwendig $)$, d. h.: $\forall x(F x \supset \forall p(p=\operatorname{daß} F x, \supset p$ ist intrinsisch form-notwendig $))$, d. h. $\forall x(F x \supset$ daß $F x$, ist intrinsisch form-notwendig), d. h. nach P2: $\forall x(F x \supset d a ß F x$, ist durch $F$ notwendig). (Äquivalenz der Hinterglieder.)

${ }^{27}$ Ang., $\exists p$ ( $p$ ist eine Erfüllung von $F$ und $p$ ist form-notwendig), also mit D4: $\exists p \exists G$ ( $p$ ist eine Erfüllung von $F$ und $p$ ist durch $G$ notwendig), also mit P7: (a) $G$ ist ein Gesetz, und (b) $F$ ist eine intensionale Teileigenschaft von $G$; also mit D13 aus (a): $\forall y G y$, und aus (b): $\forall y(G y \supset F y)$; mithin: $\forall x F x$. 


\section{Beweis:}

(i) $F$ ist ein intrinsisches Gesetz, also mit D11: $\forall y F y$ und $\forall p$ ( $p$ ist eine Erfüllung von $F \supset p$ ist durch $F$ notwendig), also: $\exists p$ ( $p$ ist eine Erfüllung von $F$, und $p$ ist durch $F$ notwendig), also mit D14: $F$ ist durch $F$ Gesetz.

(ii) $F$ ist durch $F$ Gesetz, also mit D14: $\exists p$ ( $p$ ist eine Erfüllung von $F$, und $p$ ist durch $F$ notwendig), also zum einen mit P4: $\forall p$ ( $p$ ist eine Erfüllung von $F \supset p$ ist durch $F$ notwendig), und zum anderen mit P8 (und D4): $\forall y F y$. Mithin gemäß D11: $F$ ist ein intrinsisches Gesetz.

P11 $\forall F$ : $F$ ist ein extrinsisches Gesetz $\equiv(F$ ist nicht durch $F$ Gesetz, aber $\exists G$ ( $F$ ist durch $G$ Gesetz $)$ ).

Beweis:

(i) $F$ ist ein extrinsisches Gesetz, also mit D12: $\forall y F y$ und $\forall p$ ( $p$ ist eine Erfüllung von $F \supset p$ ist nicht durch $F$ notwendig, aber es gibt ein $G$, so daß $p$ durch $G$ notwendig ist). Also zum einen: $\exists p$ ( $p$ ist eine Erfüllung von $F$, und $p$ ist nicht durch $F$ notwendig), also mit D11: $F$ ist kein intrinsisches Gesetz, also mit P10: $F$ ist nicht durch $F$ Gesetz. Also zum anderen: $\exists p(p$ ist eine Erfüllung von $F$ und $\exists G$ ( $p$ ist durch $G$ notwendig)), also gemäß D14: $\exists G$ ( $F$ ist durch $G$ Gesetz).

(ii) $F$ ist nicht durch $F$ Gesetz, aber $\exists G$ ( $F$ ist durch $G$ Gesetz); also mit D14: $\exists G \exists p$ ( $p$ ist eine Erfüllung von $F$ und $p$ ist durch $G$ notwendig). Also zum einen mit P4: $\exists G \forall p$ ( $p$ ist eine Erfüllung von $F \supset p$ ist durch $G$ notwendig), also: (1) $\forall p$ ( $p$ ist eine Erfüllung von $F \supset \exists G$ ( $p$ ist durch $G$ notwendig)). Also zum anderen mit P8: (2) $\forall y F y$. Außerdem aus der Annahme mit D14: (3) $\forall p$ ( $p$ ist eine Erfüllung von $F \supset p$ ist nicht durch $F$ notwendig). Wegen (1), (2) und (3) ergibt sich mit D12: $F$ ist ein extrinsisches Gesetz.

P12 $\forall F(F$ ist ein Gesetz $\equiv \exists G(G$ ist ein Gesetz, und $F$ ist durch $G$ Gesetz)).

Beweis:

(i) $\exists G(G$ ist ein Gesetz, und $F$ ist durch $G$ Gesetz), also mit D14: $\exists G \exists p$ ( $p$ ist eine Erfüllung von $F$, und $p$ ist durch $G$ notwendig). Also zum einen mit P8: $\forall x F x$. Also zum anderen mit P4: $\exists G \forall p$ ( $p$ ist eine Erfüllung von $F \supset p$ ist durch $G$ notwendig). Mithin wegen D13: $F$ ist ein Gesetz.

(ii) $F$ ist ein Gesetz, also mit D13: $\forall x F x$ und $\forall p$ ( $p$ ist eine Erfüllung von 
$F \supset \exists G$ (p ist durch $G$ notwendig)), also: $\exists G \exists p$ ( $p$ ist eine Erfüllung von $F$, und $p$ ist durch $G$ notwendig), also mit P7: $\exists G(G$ ist ein Gesetz und $\exists p$ ( $p$ ist eine Erfüllung von $F$, und $p$ ist durch $G$ notwendig)), also mit D14: $\exists G(G$ ist ein Gesetz, und $F$ ist durch $G$ Gesetz).

P13 $\forall F(F$ ist ein Gesetz $\supset F$ ist entweder ein intrinsisches Gesetz oder ein extrinsisches).

Beweis: Ang., $F$ ist ein Gesetz. Aus den Definitionen ist unmittelbar ersichtlich, daß kein Gesetz sowohl intrinsisch als auch extrinsisch ist. Wenn aber $F$ kein intrinsisches Gesetz ist, dann muß $F$, da ein Gesetz, ein extrinsisches Gesetz sein: Daraus, daß $F$ ein Gesetz ist, mit D13: (1) $\forall y F y$ und $\forall p$ ( $p$ ist eine Erfüllung von $F \supset$ es gibt ein $G$, so daß $p$ durch $G$ notwendig ist). Also daraus, daß $F$ kein intrinsisches Gesetz ist, mit D11: $\exists p$ ( $p$ ist eine Erfüllung von $F$, und $p$ ist nicht durch $F$ notwendig), also mit P4: $(2) \forall p$ ( $p$ ist eine Erfüllung von $F \supset p$ ist nicht durch $F$ notwendig). Aus (1) und (2) ergibt sich aufgrund von D12: $F$ ist ein extrinsisches Gesetz.

P14 $\forall p \forall F \forall G$ ( $p$ ist durch $F$ notwendig und $F$ intensionale Teileigenschaft von $G \supset p$ ist durch $G$ notwendig). [PRINZIP DER FORMVERSTÄRKUNG.]

P15 $\forall F \forall G \forall H(F$ ist durch $G$ Gesetz und $G$ durch $H$ Gesetz $\supset F$ ist durch $H$ Gesetz). [Transitivität DER FundierungsReLATION FÜR GESETZE.]

Beweis: Ang., $F$ ist durch $G$ Gesetz, und $G$ ist durch $H$ Gesetz, also mit D14: $\exists p$ ( $p$ ist eine Erfüllung von $F$, und $p$ ist durch $G$ notwendig) und $\exists p$ ( $p$ ist eine Erfüllung von $G$, und $p$ ist durch $H$ notwendig). Ang. außerdem, $F$ ist nicht durch $H$ Gesetz, also mit D14 (da $\forall y F y$ wegen der Annahme und P8 feststeht): $\forall p$ ( $p$ ist eine Erfüllung von $F \supset p$ ist nicht durch $H$ notwendig). Mithin: (1) $\exists p$ ( $p$ ist eine Erfüllung von $F$, und $p$ ist durch $G$ notwendig, und $p$ ist nicht durch $H$ notwendig), (2) $G$ ist intensionale Teileigenschaft von $H$ (wegen P7). Aber wegen P14 widersprechen sich (1) und (2).

P16 $\forall F \forall G \forall H \forall p$ ( $p$ ist eine Erfüllung von $F$ und $F$ intensionale Teileigenschaft von $G$ und $G$ Gesetz $\supset p$ ist durch $G$ notwendig). [Prinzip Der Weitergabe Der Form-Notwendigkeit; 
vgl. hierzu die Überlegungen im Anschluß an die Einführung des FundamentalPRINZIPS DER FORM-NECESSitation in Abschnitt 7.]

P17 $\forall F \forall G(F$ ist durch $G$ Gesetz $\equiv(G$ ist ein Gesetz, und $F$ ist intensionale Teileigenschaft von $G)$ ).

Beweis:

(i) $F$ ist durch $G$ Gesetz, also gemäß D14 und P7: $G$ ist ein Gesetz, und $F$ ist intensionale Teileigenschaft von $G$.

(ii) $G$ ist ein Gesetz, und $F$ ist intensionale Teileigenschaft von $G$. Also mit D13 und da $F$ intensionale Teileigenschaft von $G$ ist: $\exists p$ ( $p$ ist eine Erfüllung von $F$ ), folglich mit P16: $\exists p$ ( $p$ ist eine Erfüllung von $F$, und $p$ ist durch $G$ notwendig), also mit D14: $F$ ist durch $G$ Gesetz.

\section{Bibliographie}

Aristoteles: 1989, Metaphysik, Erster Halbband, Bücher I-VI, Griechisch und Deutsch, Übersetzung Bonitz, neubearbeitet und herausgegeben von Horst Seidl, 3. Auflage, Meiner, Hamburg.

Armstrong, David M.: 1983, What is a Law of Nature?, Cambridge University Press, Cambridge.

Armstrong, David M.: 1989, A Combinatorial Theory of Possibility, Cambridge University Press, Cambridge.

Boethius: 1949, Consolatio Philosophiae, Trost der Philosophie, Lateinisch und Deutsch, übersetzt von Eberhard Gothein, Artemis, Zürich.

Kühn, W., D. Wandschneider und U. Wolf: 1984, 'Notwendigkeit', in Joachim Ritter und Karlfried Gründer (Hrsg.), Historisches Wörterbuch der Philosophie, Bd. 6, WBG, Darmstadt, pp. 946986.

Lewis, David: 1986, On the Plurality of Worlds, Blackwell, Oxford. Lukrez: 1981, De rerum natura / Welt aus Atomen, Lateinisch und Deutsch, herausgegeben und übersetzt von Karl Büchner, Reclam, Stuttgart. 
Marc Aurel: 1990, Pros seauton - Wege zu sich selbst, Griechisch und Deutsch, herausgegeben und übersetzt von Rainer Nickel, Artemis, München / Zürich.

Meixner, Uwe: 1997, Ereignis und Substanz, Schöningh, Paderborn. Platon: 1990, Werke, Griechisch und Deutsch, Bd. 7 (Timaios, Kritias, Philebos), Übersetzung Müller und Schleiermacher, bearbeitet von Klaus Widdra, herausgegeben von Gunther Eigler, Wissenschaftliche Buchgesellschaft, Darmstadt.

Plotin: 1960, Schriften, Griechisch und Deutsch, Band Va, Übersetzung Harder, Neubearbeitung, fortgeführt von Rudolf Beutler und Willy Theiler, Meiner, Hamburg. 